Draft Version SePtember 18, 2018

Preprint typeset using $\mathrm{LAT}_{\mathrm{E}} \mathrm{X}$ style emulateapj v. 5/2/11

\title{
EXPLORING THE PROPERTIES OF MILLIARCSECOND RADIO SOURCES
}

\author{
Shuo CaO ${ }^{1}$, Marek Biesiada ${ }^{1,2}$, Xiaogang Zheng ${ }^{1}$, and Zong-Hong Zhu ${ }^{{ }^{*}}$ \\ Draft version September 18, 2018
}

\begin{abstract}
Cosmological applications of the "redshift - angular size" test require knowledge of the linear size of the "standard rod" used. In this paper, we study the properties of a large sample of 140 milliarcsecond compact radio sources with flux densities measured at $6 \mathrm{~cm}$ and $20 \mathrm{~cm}$, compiled by Gurvits et al.(1999). Using the best-fitted cosmological parameters given by Planck/WMAP9 observations, we investigate the characteristic length $l_{m}$ as well as its dependence on the source luminosity $L$ and redshift $l_{m}=l L^{\beta}(1+z)^{n}$. For the full sample, measurements of the angular size $\theta$ provide a tight constraint on the linear size parameters. We find that cosmological evolution of the linear size is small $\left(|n| \simeq 10^{-2}\right)$ and consistent with previous analysis. However, a substantial evolution of linear sizes with luminosity is still required $(\beta \simeq 0.17)$. Furthermore, similar analysis done on sub-samples defined by different source optical counterparts and different redshift ranges, seems to support the scheme of treating radio galaxies and quasars with distinct strategies. Finally, a cosmological-model-independent method is discussed to probe the properties of angular size of milliarcsecond radio quasars. Using the corrected redshift - angular size relation for quasar sample, we obtained a value of the matter density parameter, $\Omega_{m}=0.292_{-0.090}^{+0.065}$, in the spatially flat $\Lambda$ CDM cosmology.
\end{abstract}

Subject headings: quasars: general galaxies: active - radio continuum: galaxies - cosmology

\section{INTRODUCTION}

The redshift - angular size data have provided a useful method to probe cosmological parameters Guerra, Daly \& Wan 2000; Vishwakarma 2001; Lima \& Alcaniz 2002; Chen \& Ratra 2003), since this relation is directly related to the angular diameter distance. Powerful radio sources constitute a population which can be observed up to very high redshifts, reaching beyond feasible limits of supernova studies. Over a few past decades considerable advances have been made to investigate the redshift - angular size relation in radio sources for the purpose of cosmological studies, including the works of (Singal 1993; Dalv 1994; Kavser 1995; Buchalter et al. 1998; Gurvits, Kellerman \& Frev 1999; Guerra. Daly \& Wan 2000; Zhu \& Fuijimoto 2002: Podariu et al. 2003; Jackson 2004; Barai \& Wiita 2006, 2007). Up to now, redshift - angular size relation has been measured for different types of radio sources, such as extended FRIIb galaxies (Daly \& Diorgovski 2003), radio loud quasars (Buchalter et al. 1998) and radio galaxies (Guerra, Daly \& Wan 2000). In a similar spirit, by using radio observations of the Sunyaev-Zeldovich effect (SZE) together with X-ray emission of galaxy clusters, De Filippis et al. (2005); Bonamente et al. (2006) extensively explored the angular diameter distances at different redshifts.

In fact, in order to break inherent degeneracies between cosmological parameters every alternative method of restricting these parameters is desired in modern cosmology. Consequently, there have been numerous attempts to use compact radio sources for this purpose (Vishwakarma 2001; Lima \& Alcaniz 2002;

\footnotetext{
${ }^{1}$ Department of Astronomy, Beijing Normal University, 100875, Beijing, China; zhuzh@bnu.edu.cn

2 Department of Astrophysics and Cosmology, Institute of Physics, University of Silesia, Uniwersytecka 4, 40-007 Katowice, Poland
}

Zhu \& Fuiimoto 2002; Chen \& Ratra 2003). In these studies the analysis was carried out on 12 binned datapoints with 12-13 compact sources per bin. One of the major uncertainties was the typical value of the linear size $l$. In order to obtain cosmological constraints, some authors chose to fix $l$ at certain specific values (Vishwakarma 2001; Lima \& Alcaniz 2002; Zhu \& Fujimoto 2002), while Chen \& Ratra (2003) chose to include some range of values for $l$ and then marginalized over it.

It is obvious that cosmological application of the "redshift - angular size" data requires good knowledge of the linear size of the "standard rod" used. The possibility that source's linear size depends on the source luminosity and redshift should be kept in mind. In particular it still remains controversial whether compact radio sources are indeed "true" standard rods (Gurvits, Kellerman \& Frey 1999; Vishwakarma 2001). Applying the popular parametrization $l_{m}=l L^{\beta}(1+$ $z)^{n}$, Gurvits, Kellerman \& Frey (1999) claimed that by excluding sources with extreme spectral indices and low luminosities their compiled data has been minimized for the confounding by these two effects (Gurvits, Kellerman \& Frey 1999; Vishwakarma 2001). However, their results were obtained under assumption of a homogeneous, isotropic universe without cosmological constant $\left(\Omega_{\Lambda}=0\right)$. On the other hand, from a large body of recent astronomical observations, such as Union2 SNe Ia dataset (Amanullah et al. 2010), the CMB observation from the Wilkinson Microwave Anisotropy Probe (WMAP9) (Hinshaw et al. 2013), and the BAO distance ratios from the spectroscopic Sloan Digital Sky Survey (SDSS) data release7 (DR7) galaxy sample (Padmanabhan et al. 2012), no convincing evidence for deviations from the concordance $\Lambda \mathrm{CDM}$ model has been established. More recently, Planck, the thirdgeneration space mission following COBE and WMAP, 
has recently released its first cosmological results based on measurements of the CMB temperature and lensingpotential power spectra (Ade et al. 2014). All of them strongly indicate the existence of an exotic component called dark energy, which represents more than $70 \%$ of the total energy of the universe and serving as a driving force of the cosmic acceleration. Latest investigations specifically to study the properties of dark energy were carried out by Yu \& Zhu (2011); Cao, Liang \& Zhu (2011); Cao \& Zhu (2012); Cao, Covone \& Zhu (2012); Cao et al. (2012); Cao, Zhu \& Zhao (2012); Pan et al. (2012); Cao \& Liang (2013); Liao, Pan \& Zhu (2013); Cao \& Zhu (2014). Having this in mind, properties of compact radio sources should be readdressed with present angular size data and taking into account a reliable cosmology based on current precise observations.

In this paper, we will reconsider issues associated with angular sizes of radio sources under the assumption of $\Lambda$ CDM cosmological model. Specifically, we will study the characteristic length, the "angular size - luminosity" and "angular size - redshift" relations for the compact structure in quasars and radio galaxies assuming $\Lambda \mathrm{CDM}$ cosmological model as the background, which is well supported by observations. In Section 2 and 3 we briefly describe our sample, its construction and methodology of subsequent analysis. Results with the full sample and several sub-samples are presented in Section 4. Cosmological-model-independent constraints on the compact source parameters and their cosmological application are discussed in Section 5. Finally, we summarize the conclusions in Section 6 .

\section{OBSERVATIONAL DATA}

Our goal is to better constrain the parameters modeling compact radio sources, i.e. their linear size scale $l$, along with luminosity and redshift dependence of their metric linear length $l_{m}$. By "better" we mean obtained using the best currently available cosmological model. To this end, we have considered the angular size data for milliarcsecond radio sources compiled by Gurvits, Kellerman \& Frey (1999). This data set was a larger sample of sources than used by Kellermann (1993) or by Wilkinson et al. (1998) and with more complete structural data than used by Gurvits (1993, 1994).

All 330 sources included in this comprehensive compilation were imaged with very-long-baseline interferometry (VLBI) at $5 \mathrm{GHz}$ with resolution of ca. 1.5 mas. They included: 1) 79 compact radio sources associated with active galaxies and quasars considered in Kellermann (1993); 2) sources described by the Caltech - Jodrell Bank group (Xu et al. 1995; Henstock et al. 1995; Taylor et al. 1994, 1996); 3) sources discussed in other works as well as observations of high-redshift $(z>3)$ quasars (Frev et al. 1997; Paragi et al. 1998). Gurvits, Kellerman \& Frey (1999) then reduced this original compilation of 330 sources down to 145 sources, with spectral index $(-0.38 \leq \alpha \leq 0.18)$ and total luminosity $\left(L h^{2} \geq 10^{26} W \bar{W} H z^{-1}\right)$. They claimed that the former criterion helps excluding compact sources with inverted spectrum and relatively large steep spectrum, while the latter tends to minimize the possible dependence of linear size on luminosity (Gurvits, Kellerman \& Frey 1999).

Full information about all the 145 sources that remain after the aforementioned selection, can be found in Table 1 of Gurvits, Kellerman \& Frev (1999), including source coordinates, redshifts, optical counterpart, angular size, spectral index, and total flux densities at $6 \mathrm{~cm}$ or $20 \mathrm{~cm}$. Following Kellermann (1993), the characteristic angular size of each source was defined as the distance between the strongest component (referred to as the core) and the most distant component which had the peak brightness greater than or equal to $2 \%$ of the peak brightness of the core. We emphasize here, that in order to implement multi-frequency " $\theta-z$ " tests in our analysis (see the next section for details), we further restricted the final sample to 140 data points with measured flux densities at both $6 \mathrm{~cm}$ and $20 \mathrm{~cm}$. The final sample, which covers the redshift range $0.031<z<3.89$ and does not show jet-like structure for any system, contains a wide class of extragalactic objects including 112 sources identified as quasars, 18 radio galaxies, and 10 BL Lac objects (blazars).

Radio galaxies we use in this work are located within the redshift range of $0<z<0.9$. This means that, using the "redshift - angular size" test, we are able to constrain properties of active galaxies and their evolution up to $z \sim 0.9$. Main motivation of studying the milliarcsecond radio structures in quasars stems from their potential usefulness in cosmology (Kellermann 1993; Gurvits, Kellerman \& Frev 1999). Moreover, the large number of quasars is also beneficial for studying structural properties of milliarcsecond radio structures at high redshifts. Finally, inclusion of 10 sources with BL Lac objects as counterparts allows us to show that their structural properties are similar to the known quasars at $z>3$.

However, the "angular size - redshift" test requires statistically complete and well-characterized (homogeneous) sample. Because our list includes sources corresponding to different optical counterparts at different redshift as described above, so besides the full combined sample we will also consider separately seven sub-samples. Three of them are defined by optical counterparts: radio galaxies, quasars and blazars. Next subsamples are defined by restriction to four redshift ranges: $z \leq 0.5,0.5<z \leq 1.0$, $1.0<z \leq 2.0$ and $z>2.0$.

\section{METHOD}

According to Sandage (1988), the angular size-redshift relation for a rod of intrinsic length $l_{m}$ can be written as

$$
\theta(z)=\frac{l_{m}}{D_{A}(z)}
$$

where $l_{m}$ is the metric linear size, $D_{A}$ is the angular diameter distance at redshift $z$. Following the phenomenological model first proposed in Gurvits (1994) and later discussed in Gurvits, Kellerman \& Frey (1999), the projected linear size of a source is related to its luminosity $L$ and redshift $z$ as

$$
l_{m}=l\left(\frac{L}{L_{0}}\right)^{\beta}(1+z)^{n}
$$

where $l$ is the linear size scaling factor representing the apparent distribution of radio brightness from the peak down to the level of its $2 \%$ in the available sample of VLBI images. It is a parameter defined by the practical 
Table 1

Summary of constraints on the metric linear size parameters obtained with the full sample and six sub-samples (see text for definitions). Source of cosmological priors and the wavelength of measured flux are given in brackets.

\begin{tabular}{|c|c|c|c|}
\hline Sample (Cosmology+Flux density) & $l(\mathrm{pc})$ & $\beta$ & $\bar{n}$ \\
\hline Full sample (Planck $\left.+S_{6}\right)$ & $l=25.42 \pm 3.62$ & $\beta=0.169 \pm 0.025$ & $n=-0.021 \pm 0.139$ \\
\hline Full sample (WMAP9+ $\left.S_{6}\right)$ & $l=24.91 \pm 3.59$ & $\beta=0.170 \pm 0.026$ & $n=-0.009 \pm 0.141$ \\
\hline Full sample $\left(\right.$ Planck $\left.+S_{20}\right)$ & $l=27.07 \pm 3.95$ & $\beta=0.183 \pm 0.027$ & $n=-0.090 \pm 0.145$ \\
\hline Radio galaxy $\left(\right.$ Planck $\left.+S_{6}\right)$ & $l=49.55_{-20.05}^{+26.35}$ & $\beta=0.242 \pm 0.064$ & $n=0.142 \pm 0.670$ \\
\hline Quas: & $l=25.96 \pm 4.14$ & $\beta=0.203 \pm 0.034$ & $n=-0.02$ \\
\hline BL Lac (Planck & $l=20.73_{-11.78}^{+23.77}$ & $\beta=0.139 \pm 0.107$ & $n=-0.315 \pm 0.895$ \\
\hline Sub-sample $(z \leq 0.5)\left(\right.$ Planck $\left.+S_{6}\right)$ & $l=44.60 \pm 21.80$ & $\beta=0.284 \pm 0.076$ & $n=1.228 \pm 1.072$ \\
\hline Sub-sample $(0.5<z \leq 1.0)\left(\right.$ Planck $\left.+S_{6}\right)$ & $l=21.65_{-13.20}^{+21.20}$ & $\beta=0.009 \pm 0.091$ & $n=-0.335 \pm 1.115$ \\
\hline Sub-sample $(1.0<z \leq 2.0)\left(\right.$ Planck $\left.+S_{6}\right)$ & $l=63.85_{-31.85}^{+34.15}$ & $\beta=0.215 \pm 0.083$ & $n=-0.880 \pm 0.600$ \\
\hline Sub-sample $(z>2.0)\left(\right.$ Planck $\left.+S_{6}\right)$ & $l=10.49_{-8.34}^{+14.51}$ & $\beta=0.128 \pm 0.187$ & $n=0.518 \pm 0.638$ \\
\hline
\end{tabular}

limitation of dynamic range of VLBI data, i.e., a higher sensitivity of VLBI observations would enable estimates of angular sizes down to lower values of brightness, resulting in turn in a different value of the characteristic linear size (Gurvits 1994). $L_{0}$ is the normalizing luminosity taken to be equal to $10^{28} \mathrm{WHz}^{-1}$ in our analysis. Parameters $\beta$ and $n$ represent the dependence of the linear size on source luminosity and redshift, respectively. The first parameter $-\beta$ is related to physics of a compact radio emitting regions, while the parameter $n$ mimics three physical effects: (1) cosmological evolution of the linear size with redshift; (2) dependence of the linear size on the emitted frequency; and (3) an impact of sources broadening due to scattering in the propagation medium (Gurvits, Kellerman \& Frev 1999). The latter effect is not important for our sample with the lowest emitted frequency of $5 \mathrm{GHz}$ (corresponding to $z=0$ ). The distinction between the former two effects require multi-frequency $\theta-z$ tests.

The luminosity $L$ of radio sources is estimated from their measured flux density $S_{o b s}$. So the radio luminosity, assuming isotropic emission, reads:

$$
L=\frac{S_{\mathrm{obs}} 4 \pi D_{L}^{2}}{(1+z)^{1+\alpha}}
$$

where $S_{o b s}$ is the observed flux density, $D_{L}$ is the luminosity distance, and $\alpha$ is the spectral index $\left(S_{\text {obs }} \propto\right.$ $\left.\nu^{\alpha}\right)$. In general, for sources at cosmological distances, $\mathrm{k}$-correction must be applied to the spectral index $\alpha$ of the source. The angular diameter distance $D_{A}$ and the luminosity distance $D_{L}$ at redshift $z$ are related to each other through the so-called distance duality relation

$$
D_{L} / D_{A}(1+z)^{-2}=1
$$

which is a fundamental relation in observational cosmology and initiated a lot of studies, e.g. Cao \& Liang 2011; Cao \& Zhu 2011).

The above equations imply that, if we could have a reliable knowledge of cosmological model parameters which therefore allow to calculate $D_{A}$ or $D_{L}$ at different redshifts, then we would get stringent constraints on the range of parameters, $l, \beta$, and $n$ describing compact radio sources. Theoretical expression for the angular diameter distance $D_{A}(z)$ (expressed in Mpc and assuming flat FRW metric) reads

$$
D_{A}\left(z ; \Omega_{m}\right)=\frac{3000 h^{-1}}{(1+z)} \int_{0}^{z} \frac{d z^{\prime}}{E\left(z^{\prime} ; \Omega_{m}\right)}
$$

where $h$ is the dimensionless Hubble constant, $E\left(z ; \Omega_{m}\right)$ is the dimensionless expansion rate, which - in the case of flat $\Lambda$ CDM model - depends on redshift $z$ and matter density parameter $\Omega_{m}$ in the following way:

$$
E^{2}\left(z ; \Omega_{m}\right)=\Omega_{m}(1+z)^{3}+\left(1-\Omega_{m}\right) .
$$

For the purpose of our analysis, theoretical $D_{A}(z)$ has been calculated by using the best-fit matter density parameter given by Planck Collaboration: $\Omega_{m}=0.315 \pm$ 0.017 and $h=0.673 \pm 0.012$ (Ade et al. 2014). Even though Planck results are the latest ones, we also include the data from the Wilkinson Microwave Anisotropy Probe 9 year data release (WMAP9), i.e. $\Omega_{m}=0.279 \pm$ 0.025 and $h=0.700 \pm 0.022$ (Hinshaw et al. 2013). The $h=0.700$ value is also used the cosmological application of the cosmological-model-independent method is discussed in Section 5. In order to determine the parameters of compact radio structures, we preformed Monte Carlo simulations of the posterior likelihood $\mathcal{L} \sim \exp \left(-\chi^{2} / 2\right)$ using routines available in CosmoMC package. As a prior, we assumed a conservative $20 \%$ Gaussian uncertainty of the observed angular size.

\section{RESULTS AND DISCUSSIONS}

In this paper, we focused our attention on the constraints on the parameters $(l, \beta$, and $n)$ characterizing compact radio sources obtained from different samples, i.e. the full $N=140$ sample as well as several subsamples determined from different selection criteria. The results are summarized in Table 1.

\subsection{Estimates on the full sample}

As we already remarked, measured flux density at different bands could bear the information about physical conditions in active galactic nuclei - a feature common to all types of sources we used. Performing fits on the data comprising flux at $6 \mathrm{~cm}$, we obtained the following best-fit values and corresponding $1 \sigma$ (more precisely $68 \%$ 
confidence level) uncertainties

$$
\begin{aligned}
& l=25.42 \pm 3.62 \mathrm{pc}, \\
& \beta=0.169 \pm 0.025, \\
& n=-0.021 \pm 0.139 .
\end{aligned}
$$

Then, using the flux densities at $20 \mathrm{~cm}$ we obtain the following best fit

$$
\begin{aligned}
& l=27.07 \pm 3.95 \mathrm{pc}, \\
& \beta=0.183 \pm 0.027, \\
& n=-0.090 \pm 0.145 .
\end{aligned}
$$

Marginalized $1 \sigma$ and $2 \sigma$ contours of each parameter obtained at different bands are shown in Fig. 11 It is clear that the parameter degeneracies are consistent with each other, as can be seen in the contours obtained from flux densities measured at $6 \mathrm{~cm}$ and $20 \mathrm{~cm}$, respectively. Best fitted values obtained for different wavelength are different, but they agree within $1 \sigma$.

It is obvious that, for well resolved compact sources, measurements of $\theta$ provide tighter estimates of the linear size parameters $(l, \beta, n)$. More importantly, our full sample analysis has also yielded improved constraints on the meaningful physical parameters: $\beta$ and $n$. We found that best-fitted value of the parameter $n$ is a small number: $|n| \simeq 10^{-2}$, slightly negative, but $68 \%$ CI contains zero in any case i.e. our results are consistent with no evolution of $l_{m}$ with $z$. This suggests that, contrary to the case of extended radio sources, central engine powering compact radio sources is likely to be controlled by a limited number of physical parameters (mass of central black hole, accretion rate) and may therefore be less subject to evolutionary effects. On the other hand, we found that, for 140 sources satisfying luminosity selection criterion $L h^{2} \geq 10^{26} \mathrm{WHz}^{-1}$, substantial evolution of linear size with luminosity is still required. Compared with previous results obtained on the same data (Gurvits, Kellerman \& Frey 1999), our results show that, improved, more rigorous quantitative analysis supports the existence of "linear size - luminosity" relation. The conclusion that $\beta+n \geq-0.15$ given by Gurvits, Kellerman \& Frey (1999) does not contradict to the findings of the present work. The best-fit parameters of the phenomenological dependence Eq.(2) under the modern cosmological model are different from those obtained with the classical Einstein - de Sitter model used by Gurvits, Kellerman \& Frey (1999). The values of the two best-fit parameters of the phenomenological formula obtained here, namely, $\beta$ and $n$, if confirmed by future "angular size-redshift" studies, would offer additional constraints for cosmological tests based on angular sizes of extragalactic sources.

As we stressed above, the assumption of currently best available cosmological model $-\Lambda \mathrm{CDM}$ was the source of improvement concerning estimates of $l, \beta$, and $n$. Hence their values depend on the cosmological parameters used. Therefore, besides assuming flat $\Lambda$ CDM model with parameters coming from Planck observations, we also considered WMAP9 results for comparison. In this case, the

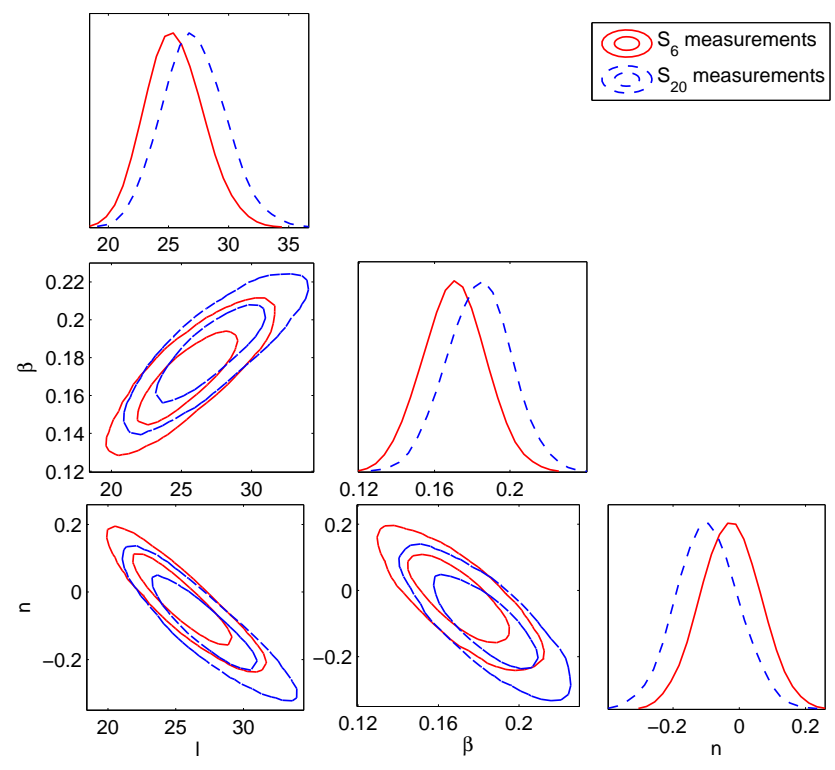

Figure 1. Constraints on compact source parameters obtained from the full sample, based on different flux density measurements at $6 \mathrm{~cm}$ and $20 \mathrm{~cm}$, respectively.

best fit is

$$
\begin{aligned}
& l=24.91 \pm 3.59 \mathrm{pc}, \\
& \beta=0.170 \pm 0.026 \\
& n=-0.009 \pm 0.141 .
\end{aligned}
$$

Marginalized probability distributions for each parameter and marginalized 2D 68\% confidence contours are presented in Fig. 2. Comparing constraints based on Planck and WMAP9 observations, we see that confidence regions of $l, \beta$, and $n$ are almost the same, hence our results and discussions presented above are robust. We remark here that, considering the WMAP9 and Planck data are consistent with the accuracy sufficient to the comparison with the " $\theta-z$ " test, it is not surprising the regression results of the " $\theta-z$ " test in combination with WMAP and Planck are compatible in the framework of $\Lambda$ CDM cosmology.

\subsection{Estimates on sub-samples}

In Table 1 and Fig. 3, 4, we show the results of fitting three parameters, $l, \beta$ and $n$ on seven sub-samples described in Section 2 ,

We note that the ranges of $l$ and $\beta$ parameters for quasars $(l=25.96 \pm 4.14 \mathrm{pc}, \beta=0.203 \pm 0.034)$ are marginally close to estimates obtained for compact structures in BL Lac objects $\left(l=20.73_{-11.78}^{+23.77} \mathrm{pc}, \beta=\right.$ $0.139 \pm 0.107)$. Rather weak dependence of the characteristic size on redshift, i.e. the range of the parameter $n$ for quasars $(n=-0.023 \pm 0.153)$ is in agreement with the estimate obtained for BL Lac sources ( $n=-0.315 \pm 0.895)$ within $1 \sigma$. On the other hand, luminosity dependence $(\beta=0.242 \pm 0.064)$ and weak redshift dependence $(n=0.142 \pm 0.670)$ are both present in radio galaxies. The best-fit values of $\beta$ and $n$ for this sub-population are significantly different from the corresponding quantities of quasars or BL Lac sources. Consequently, our results imply the existence of physical 

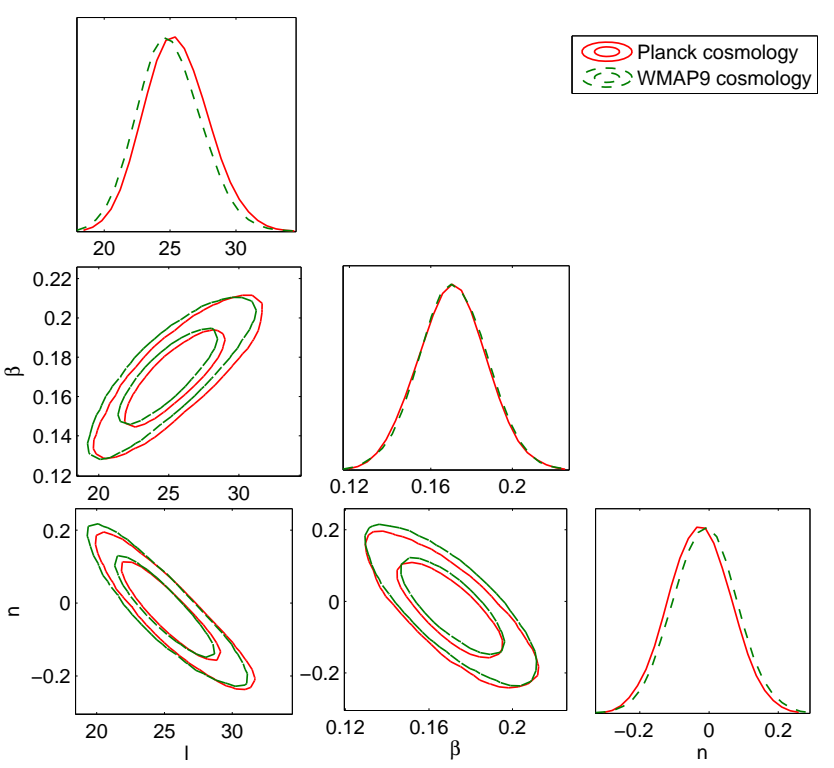

Figure 2. Constraints on compact source parameters obtained from the full sample, assuming cosmological parameters inferred from Planck and WMAP9 data.
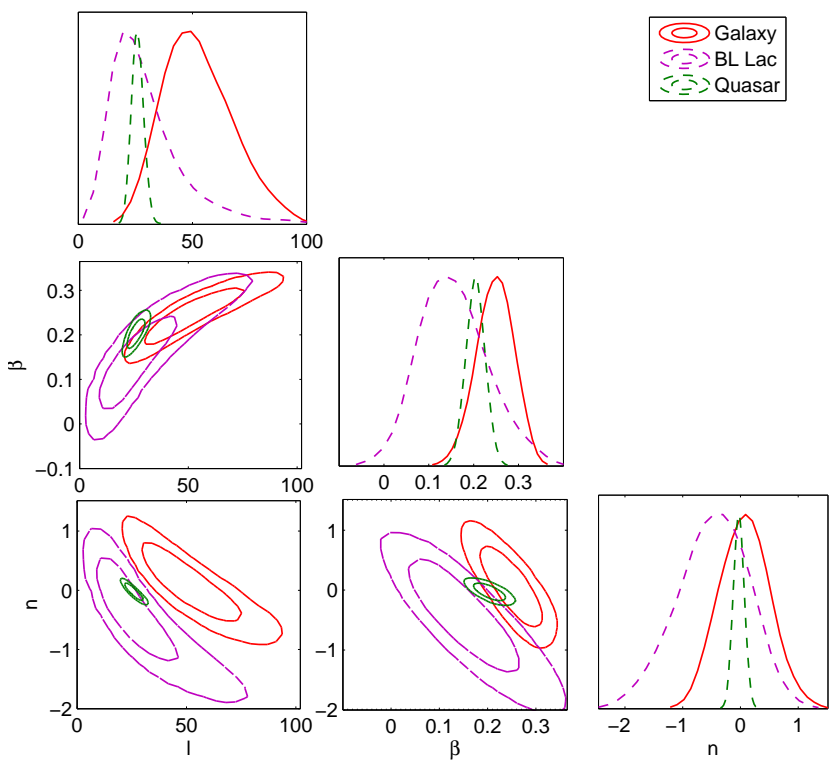

Figure 3. Constraints on compact source parameters obtained from three sub-samples with different optical counterparts.

differences between galaxies and quasars at the milliarcsecond scale. To some extent, this conclusion supports the scheme of treating radio galaxies and quasars with distinct strategies. We must keep in mind that similarity or difference in $(\beta, n)$ parameters for radio sources with different types of optical counterparts, might reveal similar or different physical processes governing the radio emission of compact structures.

This tendency could also be found in fits performed on four sub-samples with different redshift bins. From Fig. 4 we find that: (1) Constraints on all the parameters coming from the low-redshit sub-sample $(z \leq 0.5)$ are substantially different from those obtained with other

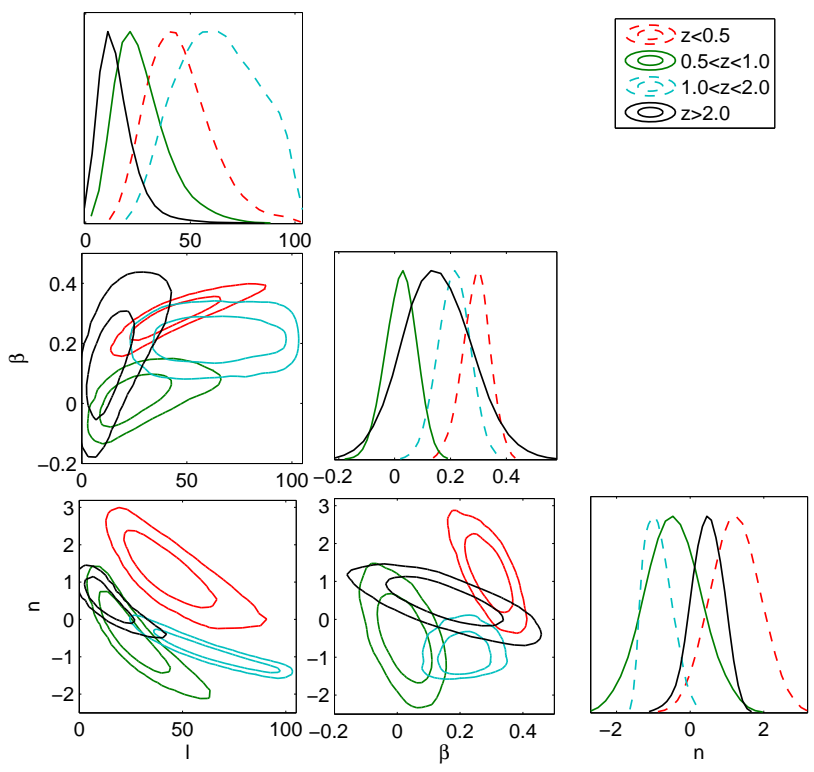

Figure 4. Constraints on compact source parameters obtained from sub-samples defined by four different redshift bins.
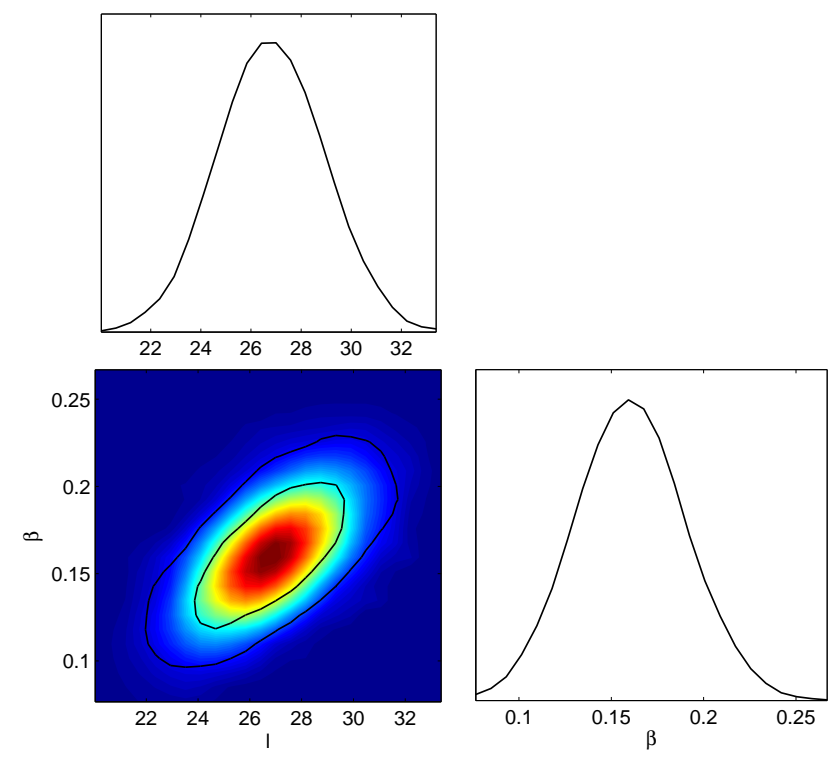

Figure 5. Constraints from the $N=26$ quasar sub-sample, with observational luminosity distance derived from Union2 SN Ia data.

sub-samples. This can be explained by the fact that that low redshift sub-sample is dominated by radio galaxies. (2) For the sub-sample with redshift range $0.5<z \leq 1.0$, the "no-evolution" model $(\beta=n=0)$ is still included within $1 \sigma$ confidence regions in $(\beta, n)$ parameter plane, whereas a substantial evolution of linear sizes with luminosity is still required for the other three sub-samples.

As we remarked above, sub-samples defined by redshift ranges are confounded with types of optical counterparts. Therefore a stratified analysis taking into account redshift ranges and the source type would be desirable. However, our sample is too small to achieve this. 


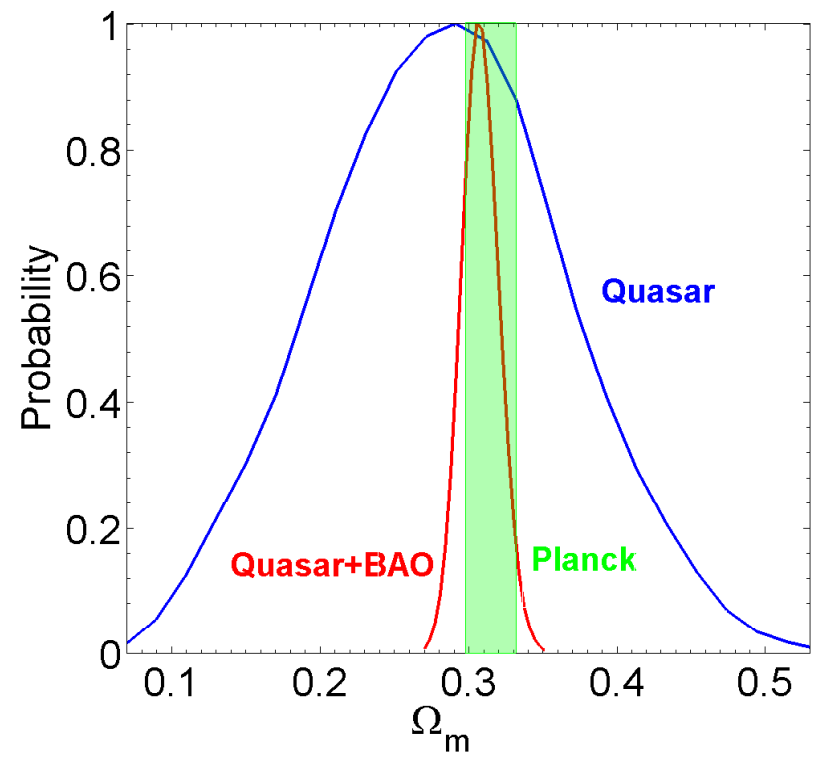

Figure 6. Cosmological fits on the $\Lambda$ CDM model form the corrected angular size - redshift relation for quasars (Blue line) and joint data sets combined with BAO observations (Red line).

5. COSMOLOGICAL-MODEL-INDEPENDENT CONSTRAINTS ON COMPACT SOURCE PARAMETERS

In the previous section, we discussed the constraints on the model parameters of compact structure in radio sources using theoretical expression for angular diameter distances and assuming best currently available $\Lambda \mathrm{CDM}$ cosmology. In this section, we propose cosmological model independent approach. Namely we derive the angular diameter distance for our radio sources from observed luminosity distance of SN Ia in the Union2 compilation (Amanullah et al. 2010). This provides us a natural way to calibrate the properties of angular size of milliarcsecond radio sources.

In order to place cosmological-model-independent constraints on $l, \beta$, and $n$, one should first perform pairwise matching of radio sources and SN Ia almost at the same redshift. Since the sample size of the SN Ia is much larger than that of the radio sources, we bin the observed $D_{A}$ (inferred from the Union2 data points) according to the following criterion: $\Delta z=\left|z_{\text {radio }}-z_{S N}\right| \leq 0.005$ (Cao \& Liang 2011). As a result we obtain a sample of 42 observational angular diameter distances $D_{A}$ derived from the supernova data covering the redshift range $0.031<z<1.34$. However, not all of them could be used in the cosmological-model-independent method. As we already discussed in Section 2 and Section 4, full $N=140$ sample is not statistically complete and homogeneous. Moreover, sources corresponding to different optical counterparts may have distinct "angular size - redshift" relation. Therefore, in cosmology independent analysis we limited ourselves to radio sources with quasars as counterparts. There are two reasons supporting this choice. First, our sample is dominated by quasars. Second, as we have seen, cosmological evolution of the linear size for quasars is very small, so we can assume $n=0$. Therefore, we finally used SN Ia to derive observational angular diameter distance for 26 quasars.

Fitting results of the compact source linear size param- eters $(l, \beta)$ are shown in Fig. [5, with the best fit

$$
\begin{gathered}
l=26.70 \pm 2.91 \mathrm{pc}, \\
\beta=0.158 \pm 0.040 .
\end{gathered}
$$

One can see that the results derived from the cosmological-model-independent analysis agree very well with the best-fitted parameters determined from theoretical cosmological distances for the $N=112$ quasar sample.

Having performed cosmological-model-independent analysis, we can consider cosmological implications of the corrected redshift - angular size relation. Using the best-fitted $l$ and $\beta$ parameters (with their $1 \sigma$ uncertainties) obtained from the model-independent analysis to the full quasar data and performing "angular size - redshift" test assuming flat $\Lambda \mathrm{CDM}$ model, we are able to get the observational constraint on the matter density parameter. The result is $\Omega_{m}=0.292_{-0.090}^{+0.065}$ and its posterior probability density function is shown in Fig. 6. We see that it is in agrement with the value obtained from Planck observations within $1 \sigma$ range around the central value. Moreover, our analysis result is fully compatible with that obtained from the previous study of peculiar velocities of galaxies, $\Omega_{m}=0.30_{-0.07}^{+0.17}$, which is the only alternative method sensitive exclusively to matter density (Feldman et al. 2003). Based on the 12 binned data-points with 12-13 compact sources per bin, it was found that Friedmann model with a vanishing $\Lambda$ is not the best-fit cosmology (Vishwakarma 2001). This result supported the necessity to include dark energy in the cosmological model. Then Lima \& Alcaniz (2002) used the same binned data to place constraints on the flat XCDM cosmology (including dark energy with constant equation of state $w$ ) with fixed physical length $l_{m} \simeq 20-30 h^{-1}$ for the radio sources. They demonstrated that the flat $\Lambda$ CDM model with $\Omega_{m}=0.20$ and $l_{m}=22.6 h^{-1}$ is the best fit to these milliarcsecond radio source data. The potential of using the same sample to study other cosmological models including dark energy with constant or time-variable equation of state was also discussed in Chen \& Ratra (2003). More recently, by applying an astrophysical model to quantify the behavior of compact radio sources as standard rods and considering possible selection effects, Jackson (2004) gave the best-fit parameter $\Omega_{m}=0.24_{-0.07}^{+0.09}$ for the flat $\Lambda \mathrm{CDM}$ model from the original data set (Gurvits 1994). We find that the constraints resulting from our analysis are consistent with the previous works. However, because we used the currently favored cosmological model and performed a cosmological model independent check, our results could be useful as hints for priors on $l, \beta$ and $n$ parameters in future cosmological studies using compact radio sources.

It has been known for some time that cosmological parameters can be more stringently determined using additional and complementary data Cao \& Zhu 2014). Therefore, we combine the compact radio sources "angular size - redshift" test with the latest data on Baryon Acoustic Oscillations (BAO). More specifically we add the BAO data from the Sloan Digital Sky Survey (SDSS) data release 7 (DR7) corresponding to $z=0.35$ (Padmanabhan et al. 2012), SDSS-III Baryon Oscillation Spectroscopic Survey (BOSS) at $z=0.57$ (Anderson et al. 2012), the clustering of WiggleZ sur- 
vey (Blake et al. 2012) at $z=0.44,0.60,0.73$ and 6dFGS survey at $z=0.10$ (Beutler et al. 2011). Likelihood distribution function for the $\Omega_{m}$ parameter in $\Lambda$ CDM model constrained by $\mathrm{BAO}$ and the compact structure in quasars is also plotted in Fig. 6. Obviously, $\Omega_{m}$ is more tightly constrained with joint data set, with the best-fit parameter $\Omega_{m}=0.308 \pm 0.019$. This also agrees with that obtained from Planck observations very well. Moreover, we find that cosmological constraint with the 112 quasars is well consistent with the joint statistical analysis.

\section{CONCLUSION AND DISCUSSION}

In this paper, we explored the properties of a sample of 140 milliarcsecond compact radio sources with measured angular sizes. Metric linear size of compact sources is usually parameterized as $l_{m}=l L^{\beta}(1+z)^{n}$. Using the best available cosmological model parameters given by the Planck/WMAP9 observations, we investigated the elements of $l_{m}$ - its characteristic length $l$ as well its dependence on the source luminosity $L$ and redshift $z$. In the full sample, we found that measurements of $\theta$ provide tighter estimate of the source linear size parameters. Small cosmological evolution of the linear size $|n| \simeq 10^{-2}$ is consistent with previous analyses, while a substantial evolution of linear sizes with luminosity is still required $(\beta \simeq 0.17)$. However, the conclusion that $\beta+n \geq-0.15$ given by Gurvits, Kellerman \& Frey (1999) does not contradict to the findings of our work.

Furthermore, by dividing the full sample into seven different sub-samples given the source redshifts and their optical counterparts, we obtain the following results: (1) The range of the parameters $\beta$ and $n$ for quasars are close to the the estimates obtained for compact structures in BL Lac sources within $1 \sigma$. (2) Both luminosity dependence and weak redshift dependence are present in radio galaxies. The best-fit values of $\beta$ and $n$ for this sub-population are significantly different from the corresponding quantities of quasars or BL Lac sources. (3) Closeness or difference of parameter values for different types of counterparts, might reveal the similar or different physical processes governing the radio emission of compact structures. This tendency could be also found from the constraints obtained with the sub-samples located at different redshift bins: (1) Constraints on the parameters with the low-redshit sub-sample $(z \leq 0.5)$ are essentially different from those obtained with other sub-samples. (2) For the sub-sample with redshift range $0.5<z \leq 1.0$, the "no-evolution" model $(\beta=n=0)$ is still included at $1 \sigma$ confidence region in the $\beta-n$ plane, whereas a substantial evolution of linear sizes with luminosity is still required for other three sub-samples.

Finally, we studied the properties of angular size of milliarcsecond radio quasars with a cosmological-modelindependent method, and then we derived the constraints (from the corrected quasar sample) on the spatially flat $\Lambda$ CDM cosmology. The obtained value of matter density parameter, $\Omega_{m}=0.292_{-0.090}^{+0.065}$, agrees very well with the previous results obtained on the same " $\theta-\mathrm{z}$ " sample and other recent astrophysical measurements including Planck observations.

Therefore, our analysis indicates that, the radio source size seems to be dependent on the source luminosity, i.e. the sources are not "true" standard rod. This is incon- sistent with their model previously discussed in the literature, in which this dependency has been minimized by discarding low values of luminosities and extreme values of spectral indices. However, in order to differentiate observational selection effect from intrinsic luminositydependence, we still need multi-frequency VLBI observations of more compact radio sources with higher sensitivity and angular resolution.

As a final remark, we point out that the sample discussed in this paper is based on VLBI images observed with various antennas configurations and techniques for image reconstruction. Our analysis potentially suffers from this systematic bias and taking it fully into account will be included in our future work. Moreover, the statistical results are obtained with VLBI images observed at frequency of $5 \mathrm{GHz}$. Since the parameter $n$ may reflect possible dependence of the linear size on the emitted frequency, multi-frequency " $\theta-z$ " tests should also be included in the future work.

The authors are grateful to the referee for very useful comments which allowed to improve the paper. This work was supported by the Ministry of Science and Technology National Basic Science Program (Project 973) under Grants Nos. 2012CB821804 and 2014CB845806, the Strategic Priority Research Program "The Emergence of Cosmological Structure" of the Chinese Academy of Sciences (No. XDB09000000), the National Natural Science Foundation of China under Grants Nos. 11373014 and 11073005, the Fundamental Research Funds for the Central Universities and Scientific Research Foundation of Beijing Normal University, and China Postdoctoral Science Foundation under grant No. 2014M550642. M.B. obtained approval of foreign talent introducing project in China and gained special fund support of foreign knowledge introducing project.

\section{REFERENCES}

Ade, P.A. R., et al. 2014, A\&A, 571, A16

Amanullah, R., et al. 2010, ApJ, 716, 712

Anderson, L., et al. 2012, arXiv:1203.6594

Barai, P., \& Wiita, P. J. 2006, MNRAS, 372, 381

Barai, P., \& Wiita, P. J. 2007, ApJ, 658, 217

Beutler, F., et al. 2011, MNRAS, 416, 3017

Blake, C., et al. 2012, MNRAS, 425, 405

Bonamente, M., et al. 2006, ApJ, 647, 25

Buchalter, A., Helfand, D. J., Becker, R. H., \& White, R. L., 1998, ApJ, 494, 503

Cao, S., Covone, G., \& Zhu, Z.-H. 2012, ApJ, 755, 31

Cao, S., \& Liang, N. 2011, RAA, 11, 1199

Cao, S., Liang, N., Zhu, Z.-H. 2011, MNRAS, 416, 1099

Cao, S., \& Liang, N. 2013, IJMPD, 22, 1350082

Cao, S., Pan, Y., Biesiada, M., Godlowski, W., \& Zhu, Z.-H. 2012, JCAP, 03, 016

Cao, S., \& Zhu, Z.-H. 2011, Science in China G: Physics and Astronomy, 54, 2260

Cao, S., \& Zhu, Z.-H. 2012, A\&A, 538, A43

Cao, S., \& Zhu, Z.-H. 2014, PRD, 90, 083006

Cao, S., Zhu, Z.-H., \& Zhao, R. 2012, PRD, 84, 023005

Chen, G., \& Ratra, B. 2003, ApJ, 582, 586

De Filippis, E., SerenoM, M., Bautz, W., Longo, G. 2005, ApJ, 625,108

Daly, R. A., 1994, ApJ, 426, 38

Daly, R. A., \& Djorgovski, S. G. 2003, ApJ, 597, 9

Feldman, H., et al. 2003, ApJ, 596, L131

Frey, S., et al. 1997, A\&A, 325, 511

Guerra, E. J., Daly, R. A., \& Wan, L. 2000, ApJ, 544, 659 
Gurvits, L. I., 1993, in Davies R. D., Booth R. S. (eds.) Sub-Arcsecond Radio Astronomy. Cambridge Univ. Press, Cambridge, p. 380

Gurvits, L. I., 1994, ApJ, 425, 442

Gurvits, L. I., Kellerman, K. I., \& Frey, S. 1999, A\&A, 342, 378

Henstock, D.R., Browne, I.W.A., Wilkinson, P. N., et al. 1995 ApJS, 100, 1

Hinshaw, G., et al. 2013, ApJS, 208, 19 arXiv:1212.5226v2]

Jackson, J. C. 2004, JCAP, 11, 7

Kayser, R. 1995, A\&A, 294, 21

Kellermann, K. I. 1993, Nature, 361, 134

Krauss, L. M., \& Schramm, D. N. 1993, ApJ, 405, L43

Lima, J. A. S., \& Alcaniz, J. S. 2002, ApJ, 566, 15

Liao, K., Pan, Y., \& Zhu, Z.-H. 2013, RAA, 13, 159

Padmanabhan, N., et al. arXiv:1202.0090

Pan, Y., et al. 2012, PLB, 718, 699

Paragi, Z., Frey, S., Gurvits, L. I., et al., 1999, Astron.Astrophys., 344,51
Podariu, S., Daly, R. A., Mory, M. P., \& Ratra, B. 2003, ApJ, 584,577

Sandage, A. 1988, ARA\&A, 26, 561

Singal, A. K. 1993, MNRAS, 263, 139

Stelmach, J. 1994, AJ, 428, 61

Taylor, G. B., Vermeulen, R. C., Pearson, T. J., et al. 1994, ApJS, 95, 345

Taylor, G. B., Vermeulen, R. C., Readhead, A.C.S., et al. 1996, ApJS, 107, 37

Vishwakarma, R. G. 2001, Classical Quantum Gravity, 18, 1159

Wilkinson, P. N., Browne, I.W.A., Alcock, D., et al. 1998, in Bremer M.N., Jackson N., Perez-Fournon I. (eds.)

Observational Cosmology with New Radio Surveys, Kluwer Acad. Publishers, Dordrecht, p. 221

Xu, W., Readhead, A.C.S., Pearson, T. J., Polatidis, A. G., Wilkinson, P. N. 1995, ApJS, 99, 297

Yu, H. \& Zhu, Z.-H. 2011, RAA, 11, 776

Zhu, Z.-H., \& Fujimoto, M. K. 2002, ApJ, 581, 1 